\title{
Cost-Effectiveness of Cabozantinib in the Second-Line Treatment of Advanced Hepatocellular Carcinoma
}

\author{
Enrique Soto-Perez-de-Celis, MD, MSc ${ }^{a, \star} ;$ Pedro N. Aguiar Jr, MD, MSc ${ }^{b, \star}$; Mónica L. Cordón, MDc; \\ Yanin Chavarri-Guerra, MD, MSc${ }^{c}$; and Gilberto de Lima Lopes Jr, MD, MBA
}

\section{ABSTRACT}

Background: Treatment options are limited for patients with advanced hepatocellular carcinoma (HCC) that progresses after treatment with sorafenib. Cabozantinib, an oral small molecule inhibitor of multiple tyrosine kinase receptors, recently showed improved overall survival (OS) compared with placebo in sorafenib-pretreated patients with advanced HCC in the CELESTIAL trial. This study assessed the costeffectiveness of cabozantinib for second-line treatment of patients with advanced HCC from a US healthcare system perspective. Patients and Methods: Cost and utility data were extracted from the CELESTIAL trial and used to determine the cost-effectiveness of cabozantinib compared with placebo plus best supportive care. The main outcome of this study was the incremental cost-effectiveness ratio (ICER), expressed as cost per quality-adjusted life year (QALY) gained by using cabozantinib compared with placebo plus best supportive care in sorafenib-pretreated HCC. Results: In the base-case analysis using data from the CELESTIAL trial, the incremental QALY and ICER were 0.067 and \$1,040,675 for cabozantinib compared with placebo and best supportive care. OS reported in the CELESTIAL trial (hazard ratio, 0.76 ; $95 \% \mathrm{Cl}, 0.63-0.92$ ) had the strongest association with the ICER. In one-way sensitivity analyses, there were no scenarios in which cabozantinib was cost-effective. In a cost-threshold analysis, cabozantinib would have to be priced at least $\$ 50$ per pill to be costeffective considering a willingness to pay of $\$ 100,000$ per QALY. Although the CELESTIAL trial demonstrated that cabozantinib improves OS compared with placebo in patients with HCC that progresses after treatment with sorafenib, our analysis shows that cabozantinib is not a cost-effective therapy in this scenario. Conclusions: At current costs, cabozantinib is not cost-effective for second-line therapy of HCC in the United States.

J Natl Compr Canc Netw 2019;17(6):669-675 doi: 10.6004/jnccn.2018.7275

${ }^{a}$ Department of Geriatrics, Instituto Nacional de Ciencias Médicas y Nutrición Salvador Zubirán, Mexico City, Mexico; ${ }^{\text {bC } C e n t r o ~ d e ~ E s t u d o s ~ e ~ P e s q u i s a ~ d e ~}$ Hematologia e Oncologia, Faculdade de Medicina do ABC, Santo André, Brazil; 'Department of Hemato-Oncology, Instituto Nacional de Ciencias Médicas y Nutrición Salvador Zubirán, Mexico City, Mexico; and dSylvester

Comprehensive Cancer Center, University of Miami, Miami, Florida.

\section{Background}

Hepatocellular carcinoma (HCC) is the most common type of primary liver cancer, the sixth most common neoplasm, and the third leading cause of cancer death worldwide. ${ }^{1,2}$ HCC incidence is highest in Eastern Asia and sub-Saharan Africa, where rates among men range from 11 to 20 per 100,000 person/years. ${ }^{1}$ In the United States, HCC incidence and mortality rates have increased, particularly among women, and 42,200 new diagnoses of primary liver and intrahepatic bile duct cancer were forecast in 2018, causing $6 \%$ of cancer-related deaths in men and $3 \%$ in women. ${ }^{3}$

HCC prognosis depends on tumor stage, degree of underlying liver dysfunction, and physical performance status. ${ }^{2}$ Curative therapies are available only for patients in whom HCC is detected at an early stage, whereas those with locally advanced or metastatic disease are eligible for palliative treatment only and have a median survival of $<1$ year. ${ }^{2,4}$ Therefore, the goal of treatment of advanced HCC is to increase survival while maintaining quality of life (QoL). ${ }^{5}$

Until 2008, no systemic treatments were available for the treatment of advanced HCC. However, improved understanding of its molecular drivers led to the development of randomized clinical trials (RCTs) of multikinase tyrosine kinase inhibitors (TKIs) for patients with HCC and conserved liver function. Two TKIs, sorafenib and regorafenib, have shown improvements in survival compared with placebo and have subsequently been approved by the FDA for use as first- and second-line therapy, respectively. ${ }^{6-8}$ In addition, another TKI, lenvatinib, was shown to be comparable to sorafenib as first-line therapy in a phase III RCT. ${ }^{9}$

The phase III CELESTIAL RCT demonstrated that the multikinase TKI cabozantinib significantly improved overall survival (OS) among patients with sorafenib-pretreated HCC and conserved liver function, with an OS of 10.2 months compared with 8 months in patients receiving placebo. ${ }^{10}$ Based on the results of CELESTIAL, a supplemental New Drug Application was filed with the FDA to expand the

See page 760 for related commentary. 
indications of cabozantinib to previously treated HCC, and its use was approved in January 2019. ${ }^{11}$

Previous studies have shown that TKIs used for the treatment of advanced HCC may not be cost-effective, which, added to their modest clinical effectiveness and their adverse side-effect profile, may limit their applicability in everyday clinical practice. ${ }^{12,13}$ This study assessed the cost-effectiveness of cabozantinib as a second-line agent in the treatment of patients with advanced HCC progressing after treatment with sorafenib from a US healthcare system perspective.

\section{Patients and Methods}

We built a decision-analytic model of patients meeting the clinical eligibility criteria from the CELESTIAL trial (incurable HCC, Child-Pugh class A liver function, progressive disease after treatment with sorafenib, and ECOG performance status of 0 or 1$).^{10}$ The model compared 2 treatment options for advanced HCC after sorafenib failure: cabozantinib or placebo (Figure 1).

We analyzed data from a US healthcare system perspective, considering drug acquisition, adverse events (AEs), and agents prescribed after progression. Other direct costs, such as for administration, monitoring, and end-of-life (EoL), were also evaluated. The utility of each health state and the disutility of each relevant $\mathrm{AE}$ were obtained from the literature.

The primary end point of this study was the incremental cost-effectiveness ratio (ICER), expressed as cost per quality-adjusted life year (QALY) gained by taking cabozantinib compared with placebo. Deterministic sensitivity analyses (DSAs) were performed to test the robustness of the results and to find factors influencing cost-effectiveness the most.

\section{Model Structure}

In the decision-analytic model (Figure 1), patients were classified into 3 mutually exclusive health states according to

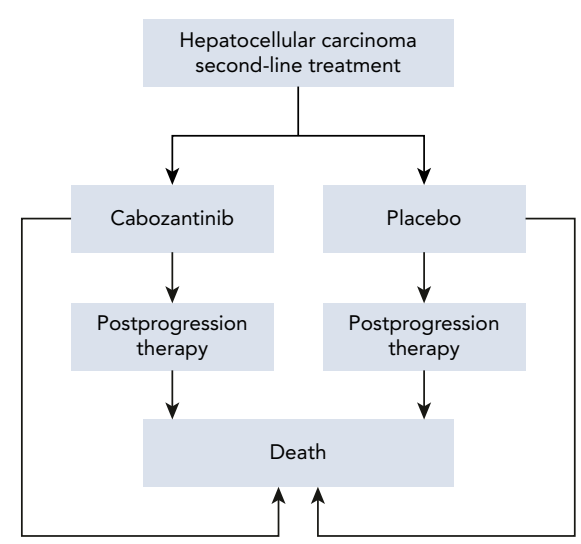

Figure 1. Decision-analytic model considering various health states. the results of the CELESTIAL trial-progression-free disease, postprogression disease, and death-and postprogression therapy was obtained from the supplemental information included in the trial..$^{10}$ All deceased patients were assumed to have received EoL care.

\section{Clinical Effectiveness and Progression Rates}

We obtained effectiveness data (mean values) from the area under the curve of progression-free survival and OS outcomes reported in the CELESTIAL RCT for both cabozantinib and placebo. ${ }^{10}$ A lifetime model was not performed considering the poor prognosis and limited life expectancy of patients with HCC that progresses on second-line therapy.

\section{Health Utilities}

Because CELESTIAL did not include a report of QoL data, we were unable to assign health utility scores for each treatment arm. ${ }^{10}$ To estimate the QoL of cabozantinibtreated patients without AEs, we extrapolated QoL data from previously published studies of cabozantinib in renal cell carcinoma (RCC). ${ }^{14}$ The QoL utilities of patients taking placebo were obtained from the placebo arm of the RESORCE trial, which studied the use of regorafenib in a similar clinical scenario as CELESTIAL. ${ }^{8}$ Because neither CELESTIAL nor RESORCE compared the QoL of patients with and without progression, we assumed that QoL was equal between patients with stable disease and those with disease progression. However, health utilities in patients with and without progression were included in the sensitivity analyses.

\section{Adverse Events}

We included the impact of the following grade 3-4 AEs (according to the CTCAE): diarrhea, palmar-plantar erythrodysesthesia, fatigue, nausea/vomiting, mucositis/ stomatitis, and hypertension. The frequency of each event was obtained from the CELESTIAL trial, ${ }^{10}$ whereas the QoL disutility of each event (according to the EuroQol 5 dimensions questionnaire [EQ-5D]) was obtained from previously published data from patients with metastatic RCC treated with targeted therapies. ${ }^{15}$ Because data on the duration of the AEs were not available, these items were included in the model as a one-off time event. For other less common AEs, we used a fixed disutility of -0.055 based on QoL data for cabozantinib in RCC. ${ }^{14}$

\section{Costs}

All costs are expressed in USD, and those that did not correspond to 2018 prices were corrected for inflation to 2018. Costs in the cabozantinib arm included cabozantinib medication costs and surveillance using imaging, laboratory tests, and clinical visits. The cost of each day of therapy with cabozantinib was determined 
from the 2018 Medicare Part D maximum allowed cost obtained using the previously published Memorial Sloan Kettering Cancer Center DrugAbacus methodology (\$566.66 per 60-mg dose). ${ }^{16,17}$ The model assumed that patients had an average time on cabozantinib of 3.8 months, according to information from CELESTIAL. ${ }^{10}$ Patients in both arms were assumed to have the same number of clinic visits, CT scans, and laboratory tests. ${ }^{18}$

Costs of AEs were calculated according to published data from patients receiving treatment of various neoplasms. ${ }^{19-24}$ In all cases, and due to the low proportion of grade 4 toxicities in the CELESTIAL trial, ${ }^{10}$ we assumed that patients were treated in an outpatient setting.

All patients who died in both arms were assumed to have received equivalent EoL care. ${ }^{24}$ The costs of postprogression therapy were calculated according to the number of patients receiving each postprogression drug and/or intervention listed in the CELESTIAL trial. ${ }^{10}$ The duration of each postprogression therapy was obtained from published phase II/III trials, and the cost was obtained from the 2018 Medicare Part B or D maximum allowed cost depending on each drug. ${ }^{8,9,16,17,25-28}$ The cost of local therapy with embolization was obtained from the 2018 Medicare Physician Fee Schedule. ${ }^{29}$

\section{Deterministic Sensitivity Analyses}

We performed several one-way DSAs to evaluate the influence of uncertainty in individual input parameters on the ICER. The 95\% CI or plausible ranges (if no confidence intervals were available) of uncertainty were considered for the most important variables. Discount rates of $10 \%, 20 \%$, and $30 \%$ on the price of cabozantinib were included in the DSA. The probability of reaching cost-effectiveness based on a willingness-to-pay threshold of $\$ 100,000$ per QALY gained was considered.

\section{Results}

Data regarding model inputs, including clinical effectiveness, frequency of AEs, costs, and QoL utilities, are shown in Table 1. Overall results of the base-case analysis are shown in Table 2. Cabozantinib provided a gain of 0.067 QALYs compared with placebo plus best supportive care. The total cost incurred with cabozantinib treatment was $\$ 109,596$ versus $\$ 39,741$ with placebo plus best supportive care. Cabozantinib was not costeffective, with an ICER of $\$ 1,040,675$ compared with placebo and best supportive care.

\section{Sensitivity Analysis}

Parameters and ranges used for the one-way DSA are included in Table 1, and all DSAs are summarized in the tornado diagram in Figure 2. The factor that had the strongest influence on the ICER was OS. When the upper limit of the $95 \%$ CI of the hazard ratio (HR) for OS was used, placebo became more cost-effective than cabozantinib, which meant that cabozantinib was dominated in this context. The strongest influence on incremental cost was progression-free survival $(95 \% \mathrm{CI})$. The next most important factors influencing cost-effectiveness were hypothetical discounts of $10 \%, 20 \%$, or $30 \%$ on the price of cabozantinib. However, there were no scenarios in which cabozantinib was cost-effective, and the ICER was always $>\$ 200,000$.

\section{Cost-Threshold Analysis}

We conducted a cost-threshold analysis to determine the cost of cabozantinib that would make it cost-effective in the studied scenario. Considering a willingness-topay of $\$ 100,000$, the maximum cost of cabozantinib that would make it cost-effective was $\$ 1,500$ per cycle ( $\$ 50$ per pill). This was related to the modest gain in QALY (0.067).

\section{Discussion}

Although the CELESTIAL trial demonstrated that cabozantinib leads to an improvement in OS compared with placebo in patients with HCC that progresses after treatment with sorafenib, our analysis shows it is not a cost-effective therapy in this scenario. Cabozantinib had an ICER of $>\$ 1$ million in the base-case analysis, and its ICERs were $>\$ 200,000$ in all one-way sensitivity analyses.

HCC usually occurs in the setting of chronic liver disease and cirrhosis, with approximately $20 \%$ of cases in the United States presenting with metastatic disease..$^{30,31}$ Systemic therapy for HCC has been recommended since the publication of the SHARP RCT, which showed that the multitargeted TKI sorafenib significantly increased OS compared with placebo (10.7 vs 7.9 months; $P<.001$ ) in patients with inoperable HCC and Child-Pugh class A cirrhosis. ${ }^{6}$ Subsequently, the phase III RESORCE RCT showed that the TKI regorafenib improved OS in patients with metastatic HCC with Child-Pugh class A cirrhosis and good performance status who experienced disease progression after treatment with sorafenib (10.6 vs 7.8 months; $P<.001$ ), leading to FDA approval of regorafenib as second-line therapy for HCC. ${ }^{8}$ The CELESTIAL RCT studied the use of cabozantinib in patients with HCC with similar characteristics as those included in RESORCE, demonstrating an improvement in OS among those who received cabozantinib compared with placebo (10.2 vs 8 months; HR, $0.76 ; 95 \% \mathrm{CI}$, $0.63-0.92 ; P=.005) .{ }^{10}$

Although these drugs prolong OS among patients with no previous therapeutic options, several criticisms have been expressed regarding their clinical use. A study 


\section{Table 1. Base-Case Model Parameters}

\section{Parameter}

Value

Reference

Clinical effectiveness

Cabozantinib

Mean OS, mo

13.95

Mean PFS, mo

6.09

Placebo

\section{Mean OS, mo}

11.19

Mean PFS, mo

HR for PFS (cabozantinib vs placebo) $(95 \% \mathrm{Cl})$

HR for OS (cabozantinib vs placebo) $(95 \% \mathrm{Cl})$

$0.76(0.63-0.92)$

Proportion of patients with grade 3-4 AEs

Cabozantinib $(n=467)$

\begin{tabular}{lc}
\hline All grade 3-4 AEs & 0.68 \\
\hline Diarrhea & 0.098 \\
\hline Palmar-plantar erythrodysesthesia & 0.169 \\
\hline Fatigue & 0.1 \\
\hline Nausea/Vomiting & 0.026 \\
\hline Hypertension & 0.16 \\
\hline Mucositis/Stomatitis & 0.034 \\
\hline Other grade 3-4 AEs & 0.58 \\
\hline Treatment-related death (grade 5) & 0.013
\end{tabular}

Placebo $(n=232)$

\begin{tabular}{ll}
\hline All grade 3-4 AEs & 0.36 \\
\hline Diarrhea & 0.016 \\
\hline Palmar-plantar erythrodysesthesia & 0 \\
\hline Fatigue & 0.042 \\
\hline Nausea/Vomiting & 0.042 \\
\hline Hypertension & 0.016 \\
\hline Mucositis/Stomatitis & 0.004 \\
\hline Other grade 3-4 AEs & 0.3 \\
\hline Treatment-related death (grade 5) & 0.004
\end{tabular}

osts, USD

\begin{tabular}{lcc}
\hline Cabozantinib, per day on drug & $\$ 566.66$ \\
\hline CT imaging & $\$ 1,539$ \\
\hline Other care & $\$ 2,871$ \\
\hline Diarrhea & $\$ 3,727$ \\
\hline Palmar-plantar erythrodysesthesia & $\$ 968$ & 17 \\
\hline Fatigue & $\$ 244$ \\
\hline Nausea/Vomiting & $\$ 2,586$ \\
\hline Mucositis/Stomatitis & $\$ 3,616$ \\
\hline Hypertension & $\$ 1,668$ \\
\hline Inpatient EoL care $(95 \% \mathrm{Cl})$ & 21 \\
\hline Total monitoring costs $(95 \% \mathrm{Cl})$ & 23 \\
\hline
\end{tabular}


Table 1. Base-Case Model Parameters (cont.)

\begin{tabular}{|c|c|c|}
\hline Parameter & Value & Reference \\
\hline Cabozantinib without $\mathrm{AEs}$ & $0.817(0.78-0.86)$ & 14 \\
\hline \multicolumn{3}{|c|}{ Disutilities from grade 3-4 AEs (with stable disease, EQ-5D) } \\
\hline Diarrhea & $0.53(0.48-0.59)$ & \multirow{5}{*}{15} \\
\hline Fatigue & $0.59(0.54-0.64)$ & \\
\hline Hypertension & $0.64(0.59-0.69)$ & \\
\hline Nausea/Vomiting & $0.54(0.48-0.59)$ & \\
\hline Mucositis & $0.53(0.47-0.57)$ & \\
\hline Other grade $3-4$ AEs (from baseline) & -0.055 & 14 \\
\hline
\end{tabular}

Abbreviations: AE, adverse events; EoL, end-of-life; EQ-5D, EuroQol 5 dimensions questionnaire; HR, hazard ratio; OS, overall survival; PFS, progression-free survival; QoL, quality-of-life.

using data from the SEER-Medicare database showed that fewer than one-third of patients with advanced HCC were able to receive first-line treatment with sorafenib and that those who did, used the drug for an average of 60 days and had a median survival of 3 months, ${ }^{32}$ which is less than the median survival of patients treated with sorafenib before inclusion in CELESTIAL (6.7 months). ${ }^{10}$ In addition, another study showed that among patients experiencing disease progression after treatment with sorafenib, only $30 \%$ were eligible for second-line therapy. ${ }^{33}$ Based on these data, the proportion of patients with HCC who would be candidates to receive secondline therapy with either regorafenib or cabozantinib has been estimated to be $<10 \%$ in everyday clinical practice. ${ }^{34}$ This calls into question the representativeness of patients included in clinical trials of second-line therapies for HCC, who are fitter and younger and have better physical performance and liver function than those seen in "real-world" settings. ${ }^{5,34}$ Another fundamental issue is the effect of these therapies on QoL and the high rates of drug-related AEs. In CELESTIAL, $68 \%$ of patients had grade 3-4 AEs and there were 6 treatment-related deaths. ${ }^{10}$ Unfortunately, the impact of cabozantinib on QoL in CELESTIAL was not reported at the same time as the clinical effectiveness results, although the protocol specified that these data were collected and analyzed using the EQ-5D questionnaire. ${ }^{10}$

From a societal perspective, studying the costeffectiveness of expensive drugs offering modest clinical benefits and having high rates of AEs is of the utmost importance. Recently published studies have shown that although both first-line sorafenib and second-line regorafenib are associated with an increase in survival, neither is cost-effective from a
Medicare perspective. ${ }^{12,13}$ In both cases, the ICER for these drugs was $>\$ 200,000$. In our study, the ICER for cabozantinib was $>\$ 1,000,000$ and it was consistently not cost-effective in all one-way sensitivity analyses.

Cost-effectiveness should not be the only factor influencing the choice of whether to recommend treatment with cabozantinib for a particular patient, and it is up to treating clinicians to use the best available evidence when making shared treatment decisions. However, oncologists should keep in mind that patients included in the CELESTIAL trial are not necessarily representative of the patient population seen in everyday clinical practice. Furthermore, although the lack of alternative therapies for patients with metastatic HCC may lead clinicians to recommend therapy with TKIs, the toxicity of these drugs must also be considered when weighing the benefits and harms of treatment. Finally, although there may be a justification for using drugs with novel mechanisms of action despite high costs, this does not seem to be the case with using pan-TKIs to treat HCC, because these drugs share common targets, and sequencing them (as done in RESORCE and CELESTIAL) may not represent a particularly novel approach.

This study has both weaknesses and strengths. Survival data were obtained from the CELESTIAL phase III clinical trial, which, as mentioned, included a population of very physically fit patients, and therefore may not reflect real-world patients. Hence, the ICER of cabozantinib may be higher than assumed in the current model. Because QoL data for patients treated with cabozantinib were not published in CELESTIAL, we extrapolated these data for patients with RCC who were receiving cabozantinib and had no AEs. ${ }^{14}$ This is 


\begin{tabular}{|c|c|c|}
\hline \multirow[b]{2}{*}{ Parameter } & \multicolumn{2}{|c|}{$\begin{array}{l}\text { Patients With HCC Progressing } \\
\text { After Treatment With Sorafenib }\end{array}$} \\
\hline & Cabozantinib & Placebo + BSC \\
\hline Treatment duration, mo & 3.8 & 2 \\
\hline \multicolumn{3}{|l|}{ Costs, USD } \\
\hline Drug & $\$ 64,599$ & $\$ 0$ \\
\hline AEs & $\$ 1,137$ & $\$ 207$ \\
\hline Postprogression therapies & $\$ 35,290$ & $\$ 30,702$ \\
\hline EoL care & $\$ 5,185$ & $\$ 5,448$ \\
\hline Monitoring & $\$ 3,384$ & $\$ 3,384$ \\
\hline Total cost & $\$ 109,596$ & $\$ 39,741$ \\
\hline \multicolumn{3}{|l|}{ Clinical effectiveness, mo } \\
\hline Mean PFS & 6.09 & 2.32 \\
\hline Mean postprogression survival & 7.86 & 8.87 \\
\hline Mean OS & 13.95 & 11.19 \\
\hline \multicolumn{3}{|l|}{ Utilities (EQ-5D) } \\
\hline Utility & 0.92 & 0.72 \\
\hline AEs & -0.17 & -0.04 \\
\hline QALY & 0.75 & 0.68 \\
\hline QALY gain & \multicolumn{2}{|c|}{0.067} \\
\hline ICER & \multicolumn{2}{|c|}{$\$ 1,040,675$} \\
\hline LYG & 1.16 & 0.93 \\
\hline Incremental LYG & \multicolumn{2}{|c|}{0.23} \\
\hline Cost per incremental LYG, USD & \multicolumn{2}{|c|}{$\$ 303,716$} \\
\hline
\end{tabular}

Abbreviations: $A E$, adverse event; $B S C$, best supportive care; EoL, endof-life; EQ-5D, EuroQol 5 dimensions questionnaire; HCC, hepatocellular carcinoma; ICER, incremental cost-effectiveness ratio; LYG, life-years gained; OS, overall survival; PFS, progression-free survival; $Q A L Y$, quality-adjusted life-year.

appropriate because we assumed that in both scenarios patients had stable disease, and the utility data were very similar to those reported for patients without AEs in the RESORCE trial of regorafenib. In addition, because data regarding the effect of progressive disease on QoL were not available, we assumed that disutilities were equal in patients with stable disease and progressive disease, which may not reflect the full range of QoL changes associated with disease progression. We were also unable to obtain the characteristics and duration of AE disutilities for patients with HCC receiving treatment with TKIs, so the data were extrapolated from studies conducted in patients with RCC. ${ }^{15}$ As many of those patients were undergoing treatment with TKIs, we consider this a correct approach that might be more appropriate than one extrapolating disutilities from patients with breast cancer, as in previous studies exploring the costeffectiveness of second-line treatments of HCC. ${ }^{13}$ One of the specific strengths of this analysis is that we considered the cost of postprogression therapies, many

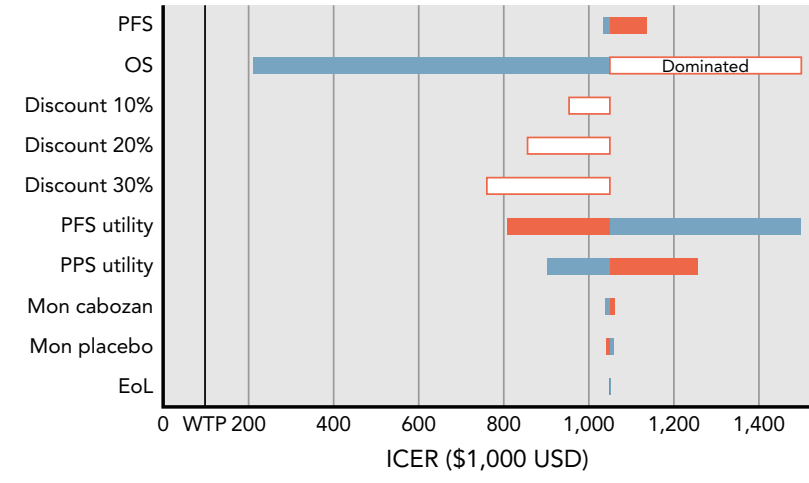

Figure 2. Tornado diagram showing one-way deterministic sensitivity analyses. Modifications in the ICER occur as model parameters are varied over prespecified ranges. Red bars represent the upper values of the parameters, whereas blue bars represent the lower values. White bars represent price discounts. "Dominated" bars mean that considering the upper parameter values made placebo more effective than cabozantinib.

Abbreviations: Cabozan, cabozantinib; EoL, end-of-life; ICER, incremental cost-effectiveness ratio; Mon, monitoring; OS, overall survival (with cabozantinib); PFS, progression-free survival (with cabozantinib); PPS, postprogression survival; WTP, willingness to pay.

of which are currently being used in clinical practice in the United States. Finally, it is important to mention that this study only accounts for the perspective of the Medicare program and that the results may differ when other perspectives are used.

\section{Conclusions}

Regardless of its modest clinical effectiveness in the context of an RCT, cabozantinib is not cost-effective for the treatment of patients with HCC that progresses after first-line therapy with sorafenib. The high cost of cabozantinib, combined with its AE profile, suggests that it may represent a low-value therapeutic alternative in this setting. We strongly believe that novel therapeutic strategies are needed to improve the outcomes of patients with advanced HCC and that future studies should strive to include a patient population that is more representative of everyday clinical practice.

Submitted August 29, 2018; accepted for publication January 16, 2019.

Author contributions: Study concept: Soto-Perez-de-Celis, Aguiar, Cordón, Lopes. Data curation: Soto-Perez-de-Celis, Aguiar, Cordón Formal analysis: Aguiar. Investigation: All authors. Methodology: Soto-Perez-deCelis, Aguiar. Manuscript preparation: All authors. Critical revision: All authors.

Disclosures: The authors have not received any financial consideration from any person or organization to support the preparation, analysis, results, or discussion of this article.

Correspondence: Gilberto de Lima Lopes Jr, MD, MBA, Sylvester Comprehensive Cancer Center, University of Miami, 1120 NW 14th Street, Suite 650J, Miami, FL 33135. Email: glopes@med.miami.edu 


\section{References}

1. Ferlay J, Soerjomataram I, Ervik M, et al. GLOBOCAN 2012 v1.0, cancer incidence and mortality worldwide: IARC CancerBase No. 11. Available at: http://globocan.iarc.fr. Accessed August 12, 2018

2. Forner A, Reig M, Bruix J. Hepatocellular carcinoma. Lancet 2018;391: 1301-1314.

3. Siegel RL, Miller KD, Jemal A. Cancer statistics, 2018. CA Cancer J Clin 2018;68:7-30

4. Lopez PM, Villanueva A, Llovet JM. Systematic review: evidence-based management of hepatocellular carcinoma-an updated analysis of randomized controlled trials. Aliment Pharmacol Ther 2006;23:1535-1547.

5. Marrero JA, Kulik LM, Sirlin CB, et al. Diagnosis, staging, and management of hepatocellular carcinoma: 2018 practice guidance by the American Association for the Study of Liver Diseases. Hepatology 2018; 68:723-750.

6. Llovet JM, Ricci S, Mazzaferro V, et al. Sorafenib in advanced hepatocellular carcinoma. N Engl J Med 2008;359:378-390.

7. Cheng $A L$, Kang $Y K$, Chen Z, et al. Efficacy and safety of sorafenib in patients in the Asia-Pacific region with advanced hepatocellular carcinoma: a phase III randomised, double-blind, placebo-controlled trial. Lancet Oncol 2009;10:25-34.

8. Bruix J, Qin S, Merle P, et al. Regorafenib for patients with hepatocellular carcinoma who progressed on sorafenib treatment (RESORCE): a randomised, double-blind, placebo-controlled, phase 3 trial. Lancet 2017;389:56-66.

9. Kudo M, Finn RS, Qin S, et al. Lenvatinib versus sorafenib in first-line treatment of patients with unresectable hepatocellular carcinoma: a randomised phase 3 non-inferiority trial. Lancet 2018;391:1163-1173.

10. Abou-Alfa GK, Meyer T, Cheng AL, et al. Cabozantinib in patients with advanced and progressing hepatocellular carcinoma. N Engl J Med 2018; 379:54-63.

11. The ASCO Post. FDA accepts supplemental new drug application for cabozantinib in previously treated advanced hepatocellular carcinoma. Available at: http://www.ascopost.com/News/58887. Published May 30, 2018. Accessed August 12, 2018.

12. Parikh ND, Marshall VD, Singal AG, et al. Survival and cost-effectiveness of sorafenib therapy in advanced hepatocellular carcinoma: an analysis of the SEER-Medicare database. Hepatology 2017;65:122-133.

13. Parikh ND, Singal AG, Hutton DW. Cost-effectiveness of regorafenib as second-line therapy for patients with advanced hepatocellular carcinoma. Cancer 2017;123:3725-3731.

14. Meng J, Lister J, Vataire AL, et al. Cost-effectiveness comparison of cabozantinib with everolimus, axitinib, and nivolumab in the treatment of advanced renal cell carcinoma following the failure of prior therapy in England. Clinicoecon Outcomes Res 2018;10:243-250.

15. Swinburn $P$, Lloyd $A$, Nathan $P$, et al. Elicitation of health state utilities in metastatic renal cell carcinoma. Curr Med Res Opin 2010;26:1091-1096.

16. Memorial Sloan Kettering Cancer Center Drug Pricing Lab. Drug abacus methods. Available at: https://drugpricinglab.org/tools/drug-abacus/ methods/. Accessed August 12, 2018.

17. Medicare.gov. Medicare plan finder. Available at: https://www.medicare. gov/find-a-plan/questions/home.aspx. Accessed August 12, 2018.

18. Carr BI, Carroll S, Muszbek N, et al. Economic evaluation of sorafenib in unresectable hepatocellular carcinoma. J Gastroenterol Hepatol 2010; 25:1739-1746.
19. Hagiwara M, Borker R, Oster G. Economic burden of adverse events in patients with metastatic renal cell carcinoma. Clin Ther 2013;35: 1955-1963.

20. Borovicka JH, Calahan C, Gandhi M, et al. Economic burden of dermatologic adverse events induced by molecularly targeted cancer agents. Arch Dermatol 2011;147:1403-1409.

21. Ting J, Tien Ho P, Xiang $P$, et al. Cost-effectiveness and value of information of erlotinib, afatinib, and cisplatin-pemetrexed for first-line treatment of advanced EGFR mutation-positive nonsmall-cell lung cancer in the United States. Value Health 2015;18: 774-782.

22. Elting LS, Shih YC. The economic burden of supportive care of cancer patients. Support Care Cancer 2004;12:219-226.

23. Janke AT, McNaughton CD, Levy PD. Incidence and cost of hypertensive emergencies in United States emergency departments. Ann Emerg Med 2015;66(Suppl):S68

24. May P, Normand C, Cassel JB, et al. Economics of palliative care for hospitalized adults with serious illness: a meta-analysis. JAMA Intern Med 2018;178:820-829

25. Yezefski T, Le D, Chen L, et al. Comparison of chemotherapy use, cost, and survival in patients with metastatic colorectal cancer in Western Washington and British Columbia [abstract]. J Clin Oncol 2018; 36(Suppl):Abstract LBA3579.

26. Qin S, Bai Y, Lim HY, et al. Randomized, multicenter, open-label study of oxaliplatin plus fluorouracil/leucovorin versus doxorubicin as palliative chemotherapy in patients with advanced hepatocellular carcinoma from Asia. J Clin Oncol 2013;31:3501-3508.

27. Centers for Medicare \& Medicaid Services. Medicare ASP drug pricing files. Available at: https://www.cms.gov/apps/ama/license.asp?file=/ Medicare/Medicare-Fee-for-Service-Part-B-Drugs/McrPartBDrugAvgSalesPrice/ Downloads/2018-July-ASP-Pricing-File.zip. Accessed August 12, 2018.

28. Zhu AX, Finn RS, Edeline J, et al. Pembrolizumab in patients with advanced hepatocellular carcinoma previously treated with sorafenib (KEYNOTE-224): a non-randomised, open-label phase 2 trial. Lancet Oncol 2018;19:940-952.

29. Centers for Medicare \& Medicaid Services. Physician Fee Schedule. CY 2018 Physician Fee Schedule Final Rule. Available at: https://www.cms. gov/Medicare/Medicare-Fee-for-Service-Payment/PhysicianFeeSched/. Accessed August 12, 2018

30. Noone AM, Howlader N, Krapcho M, et al, eds. SEER Cancer Statistics Review, 1975-2015, National Cancer Institute. Bethesda, MD. Available at: https://seer.cancer.gov/csr/1975_2015/, based on November 2017 SEER data submission, posted to the SEER web site, April 2008. Accessed August 12, 2018.

31. Rich NE, Hester $\mathrm{C}$, Odewole $\mathrm{M}$, et al. Racial and ethnic differences in presentation and outcomes of hepatocellular carcinoma. Clin Gastroenterol Hepatol 2018;17:551-559.

32. Sanoff HK, Chang $Y$, Lund JL, et al. Sorafenib effectiveness in advanced hepatocellular carcinoma. Oncologist 2016;21:1113-1120.

33. Lee IC, Chen $Y T$, Chao $Y$, et al. Determinants of survival after sorafenib failure in patients with BCLC-C hepatocellular carcinoma in real-world practice. Medicine (Baltimore) 2015;94:e688.

34. Gyawali B, Prasad V. Me too-drugs with limited benefits-the tale of regorafenib for HCC. Nat Rev Clin Oncol 2017;14:653-654. 